\title{
Greenhouse effect from the point of view of radiative transfer
}

\author{
S. Barcza $^{1}$ (D)
}

Received: 25 February 2016/Accepted: 19 September 2016/Published online: 28 September 2016

(C) Akadémiai Kiadó 2016

\begin{abstract}
Radiative power balance of a planet in the solar system is delineated. The terrestrial powers are transformed to average global flux in an effective atmospheric column approximation, its components are delineated. The estimated and measured secular changes of the average global flux are compared to the fluxes derived from the StefanBoltzmann law using the observed global annual temperatures in the decades between 1880 and 2010. The conclusion of this procedure is that the radiative contribution of the greenhouse gas $\mathrm{CO}_{2}$ is some $21 \pm 7 \%$ to the observed global warming from the end of the nineteenth century excluding the feedback mechanisms playing determining role in the climate system. Stationary radiative flux transfer is treated in an air column as a function of the column density of the absorbent. Upper and lower limit of radiative forcing is given by assuming true absorption and coherent scatter of the monochromatic radiation. An integral formula is given for the outgoing long wave radiation as a function of column density of the components of greenhouse gases.
\end{abstract}

Keywords Greenhouse effect · Total solar irradiation · Radiative fluxes

\section{Introduction}

Terrestrial atmospheric greenhouse effect, and its eventual change by the increment of the concentration of greenhouse gases, resulting in a global forcing, is a topic of many observational and theoretical papers. The present paper contributes to this field by presenting a solution to the problem of the greenhouse effect, adapted from the physics of stellar atmospheres (Mihalas 1978). The main difference in comparison to the stellar case

\section{S. Barcza}

barcza@konkoly.hu

1 Research Centre for Astronomy and Earth Sciences, Geodetic and Geophysical Institute, Konkoly Observatory, Hungarian Academy of Sciences, Sopron, Hungary 
is that the conditions are far from local thermodynamic equilibrium (LTE). The radiation has an asymmetry in the sense that the incoming downward radiation is in the optical domain of wavelengths which is partly reflected, partly absorbed and converted to the emitted upward infrared radiation leaving finally the atmosphere. Furthermore, an appropriate time averaging is necessary because the radiation field is variable in a planetary atmosphere, there are diurnal and seasonal variations.

The goal of the paper is to reveal long term changes in the radiative properties of a planetary atmosphere, which are significant for climatic considerations. The elementary task is pursued here to solve the stationary radiative transfer problem in the case of an effective air column (EAC) or water column. The change of the emerging flux is derived as a function of variable column density of the greenhouse gases. This task is relatively simple, it is a primary component in involved global climate models. It can be separated from the problem to synthetize an overall atmospheric model which would treat the hydrodynamical, radiative, chemical and phase changes and take into account the geographical differences resulting finally in a climate change. This coupled problem is beyond the scope of the present paper because oceans, solid surface and atmosphere compose a very complex system and have a large number of degrees of freedom to store and emit the energy absorbed from the incoming flux. Furthermore, internal non-radiative degrees of freedom exist among which energy transfer takes place under conditions far from LTE. These factors can result in climate fluctuations by feedback mechanisms having much larger characteristic times compared to the radiative processes which are discussed in the present paper and result in changes over a few days time scale.

Large amount of temperature measurements are available at the terrestrial surface and in the atmosphere. Homogenizing these data and to derive appropriate global averages is an ambiguous task. Additional problem is that the temperature is an intensive quantity of thermodynamics, that is, temperatures are only statistical data for a quantitative measurement of the greenhouse effect. The flux and power are extensive quantities of thermodynamics and atmospheric physics. The main goal of this paper to calculate temperatures from propagation, balance and conversion of the radiative flux. The discussions are restricted to radiative processes, the condition of the convective instability is not discussed. The crucial quantities are integrated power and flux of incoming and outgoing electromagnetic radiation, the computation of specific intensity is not necessary.

The power and flux describe the variable absorbed or emitted electromagnetic radiation over time intervals extending from minutes to centuries. The time interval must be sufficiently long if the greenhouse effect is to be treated. It must not be forgotten that the units of power and flux, watts and watts/surface, rely for seconds, averaging these quantities for a whole planet and for time intervals century or longer must be done carefully.

The paper is organized as follows. Section 2 defines the greenhouse effect, introduces the powers and fluxes which are the main quantities, the units $\mathrm{W}$ and $\mathrm{Wm}^{-2}$ are used for them. The problem of converting them into the more familiar quantity of temperature is indicated. Section 3 reviews the total solar irradiation and its variability. Section 4 is devoted to derive the flux propagation, the connection of flux with albedo and greenhouse effect is enlightened. The radiative contribution of the increasing concentration of the atmospheric greenhouse gases for the present day global forcing is estimated in Sect. 5. The results are discussed in Sect. 6, the conclusions are drawn in Sect. 7. 


\section{The greenhouse effect, empirical approach}

An empirical definition of the local greenhouse effect at a geographical point was given by (Raval and Ramanathan 1989) in terms of the flux $\mathcal{F}_{\mathrm{G}}$

$$
\mathcal{F}_{\mathrm{G}}=\mathcal{F}_{\text {surf }}-\mathcal{F}_{\text {OLR }}
$$

where $\mathcal{F}_{\text {surf }}$ and $\mathcal{F}_{\text {OLR }}$ are the integrated flux emitted by the surface and the outgoing longwave flux at the top of the atmosphere (TOA), respectively. $\mathcal{F}_{\mathrm{G}}$ is the trapped flux by the atmosphere, it warms the atmosphere. Of course, these are integrated fluxes for the infrared wavelengths.

The source of $\mathcal{F}_{\text {surf }}$ is the total solar irradiation (TSI)

$$
\mathcal{F}_{\mathrm{S}}=1361.5 \mathrm{Wm}^{-2} \mathrm{~d}^{-2}
$$

(Solanki and Unruh 2013) where $d$ is the distance of the planet to the Sun in astronomical units $\left(1 \mathrm{au}=1.4962 \times 10^{8} \mathrm{~km}\right.$.) $\mathcal{F}_{\mathrm{S}}$ enters in the atmosphere under different angles depending on geographical position and rotation, it is disseminated over the whole Earth giving the averaged flux $\mathcal{F}_{\mathrm{S}} / 4=340.38 \mathrm{Wm}^{-2}$. This approximation treats the atmosphere as an EAC. The theoretically computed fluxes of an EAC can be compared to averaged fluxes over different geographical locations if they were constructed by carefully averaging measured and theoretically computed atmospheric fluxes, for a part of the surface or for the whole Earth. The EAC model is applied for the whole Earth in many papers like e.g. (Trenberth et al. 2009) etc. Definition (1) of $\mathcal{F}_{\mathrm{G}}$ must be supplemented by the contribution from the flux transfer between the optical irradiance and infrared flux which propagates in the atmosphere. The eventual infrared flux from the surface can easily be introduced in (1) by an additive term to $\mathcal{F}_{\text {surf }}$.

The power $W_{\mathrm{S}}=\pi R^{2} \mathcal{F}_{\mathrm{S}}$ is available for a planet of radius $R$ to cover the needed power of meteorological, climatological processes in the atmosphere, oceans and at the solid surface. $W_{\mathrm{S}}=1.723 \times 10^{17} \mathrm{~W}$ is available for the whole Earth at $d=1$. Radiation is only possibility to exchange power with the interplanetary environment since the particle irradiation of the Sun is negligible in comparison to that of the incoming electromagnetic radiation. The power balance of the Earth (or a planet) is expressed at the TOA by

$$
W_{\mathrm{S}}=W_{\mathrm{O}}+W_{\mathrm{OLR}}-W_{\mathrm{I}}
$$

where $W_{\mathrm{O}}, W_{\mathrm{OLR}}$ are the reflected, absorbed (and reemitted) powers in the optical and infrared wavelengths, respectively. $W_{\mathrm{I}}$ was added for the sake of completeness, it is the power released by the Earth itself, e.g. from the radioactive internal heat production $4.42 \times 10^{13} \mathrm{~W}$ (Lowrie 2007). It must be noted for orientation that the averaged power release from the global industrial energy production $\approx 5.6 \times 10^{20} \mathrm{~J} /$ year (IEA 2014) contributes to $W_{\mathrm{I}} 1.78 \times 10^{13} \mathrm{~W} \approx 10^{-4} W_{\mathrm{S}}$. However, this component of $W_{\mathrm{I}}$ is negligible for the Earth in comparison to the other powers in (3), but the $\mathrm{O}\left(10^{-4} W_{\mathrm{S}}\right)$ is a few percent of the net down value in the CERES period which can be the suspected imbalance leading to the recent warming of the climate (Trenberth et al. 2009, 2014).

The dissipation produces OLR from all meteorological, climatic processes. The greenhouse effect is stationary if all components are constant in (3). Equation (3) takes the form in terms of time averaged integrated fluxes

$$
\frac{1}{4} \mathcal{F}_{\mathrm{S}}-\mathcal{F}_{\mathrm{O}}-\mathcal{F}_{\mathrm{OLR}}+\mathcal{F}_{\mathrm{I}}=0
$$


at the TOA which is defined by the zero optical depth $\tau=0$ for all wavelengths and $\mathcal{F}_{\text {OLR }}$ contains now the infrared flux converted from the optical one before reaching the surface. Equation (4) can be extended to any optical depth and it renders possible to treat quantitatively the greenhouse effect in the EAC approximation.

To determine the effect of the trapped flux $\mathcal{F}_{\mathrm{G}}$ on the climate is equivalent to solve the problem of the propagation and absorption of radiation in an air column and to convert the flux to local temperature by taking into account the interaction of the radiation and atmospheric matter. This interaction takes place among conditions which are far from LTE. The irradiation by the Sun at a planet differs considerably from the Planck-distribution of flux

$$
\mathcal{F}_{\lambda}^{(+)}(T)=\frac{2 \pi h c^{2}}{\lambda^{5}(\exp \{h c / k T \lambda\}-1)}
$$

where $T=T_{\mathrm{e}, \text { Sun }}=5780 \mathrm{~K}$ and $\mathcal{F}_{\lambda}^{(+)}(T)$ must be multiplied by the dilution factor $d^{-2}$. $(+)$ indicates that the flux is emitted in the half hemisphere above the surface of a celestial body. The emitted radiation of a terrestrial ocean follows the Planck-distribution within 1 $\%$ at infrared wavelengths (Raval and Ramanathan 1989; Beuttner and Kern 1965) if $T \approx T_{\text {surf }}$ is introduced in (5).

The inversion of the Stefan-Boltzmann law allows to order an effective temperature to a flux $\mathcal{F}^{(+)}$:

$$
T_{\mathrm{e}}=\left(\frac{\mathcal{F}^{(+)}}{\sigma}\right)^{1 / 4}, \quad \sigma=5.672 \times 10^{-8} \frac{\mathrm{W}}{\mathrm{m}^{2} \mathrm{~T}^{4}} .
$$

It must be emphasized that $T_{\mathrm{e}}$ is an effective, not a local temperature. It is rather a model parameter of the EAS because the temperature in an air column is height dependent. The $T_{\mathrm{e}}$ at the TOA for planets of the solar system and Saturn moon Titan is shown in Table 1 in comparison to the empirically measured temperatures in the atmosphere or at the surface of these celestial bodies. A strong forcing is obvious in the atmosphere of Venus which is much hotter than the temperature expected by the solar irradiation, in line with the StefanBoltzmann law. The atmosphere of the Earth and perhaps of Titan produces a slight forcing while the forcing at the TOA of the other planets, - that is the greenhouse effect-is almost negligible.

\section{The total solar irradiation (TSI)}

The TSI $\left(=\mathcal{F}_{\mathrm{S}}\right)$ is not constant. The observed variability originating from the Sun between 1979 and 2010 is reported in (Solanki and Unruh 2013). The time scales of solar

Table $1 \quad T_{\mathrm{e}}=\left(\frac{1}{4} \mathcal{F}_{\mathrm{S}} / \sigma\right)^{1 / 4}$ and empirical surface temperatures $T_{\text {surf }}$ in the solar system

* TOA temperatures

\begin{tabular}{llll}
\hline & $d[\mathrm{au}]$ & $T_{\mathrm{e}}$ & $T_{\text {surf }}$ \\
\hline Venus & 0.72 & 328 & $\approx 730$ \\
Earth & 1 & 278 & $\approx 287$ \\
Mars & 1.52 & 226 & $\approx 218$ \\
Jupiter & 5.20 & 122 & $\approx 120^{*}$ \\
Saturn & 9.55 & 90 & $\approx 88^{*}$ \\
Titan & 9.55 & 90 & $\approx 95$ \\
\hline
\end{tabular}


oscillations, solar magnetic cycle, solar cycle and secular changes vary from 5 min to $10^{5}-10^{6}$ years. The amplitudes are known from observations over decade time scale, they are below $10^{-3} \mathcal{F}_{\mathrm{S}}$ for the oscillations and the cycles. The small downward drift of the observed TSI $\left(<10^{-4}\right.$ over decade time scale) and the long term secular changes (which are known only from theoretical considerations with large uncertainty) seem to be irrelevant for the observed present day forcing (Solanki et al. 2013, upper panel in Fig. 1). For climatological considerations the conclusion is that the averaged value of the TSI during the few decades of the observations can be regarded as constant.

A critical remark is appropriate on the observed TSI. The ground based observations resulted in $1368 \mathrm{~d}^{-2} \mathrm{Wm}^{-2}$ a half century ago (Unsöld 1968). The results of earth radiation budget experiment (ERBE) and clouds and the earth's radiant energy system (CERES) for the TSI are $1367 \mathrm{~d}^{-2} \mathrm{Wm}^{-2}$ (1985-1989, minimal solar activity) and $1365 \mathrm{~d}^{-2} \mathrm{Wm}^{-2}$, (2000-2004, maximal solar activity), respectively. The result of a Japanese reanalysis is $1356 \mathrm{~d}^{-2} \mathrm{Wm}^{-2}$, while the average over 1979-2010 is $1361.5 \mathrm{~d}^{-2} \mathrm{Wm}^{-2}$. An eventual explanation of the forcing by the TSI would require an ascending TSI $\approx 18 \mathrm{Wm}^{-2}$ (for $1880-2010$ ) or $\approx 12 \mathrm{Wm}^{-2}$ between 1979 and 2010 , respectively. Changes of this size are definitely not present in the observational results. This somewhat controversial situation is plotted in terms of $\frac{1}{4} \mathcal{F}_{\mathrm{S}}$, right part of Fig. 1, upper panel.
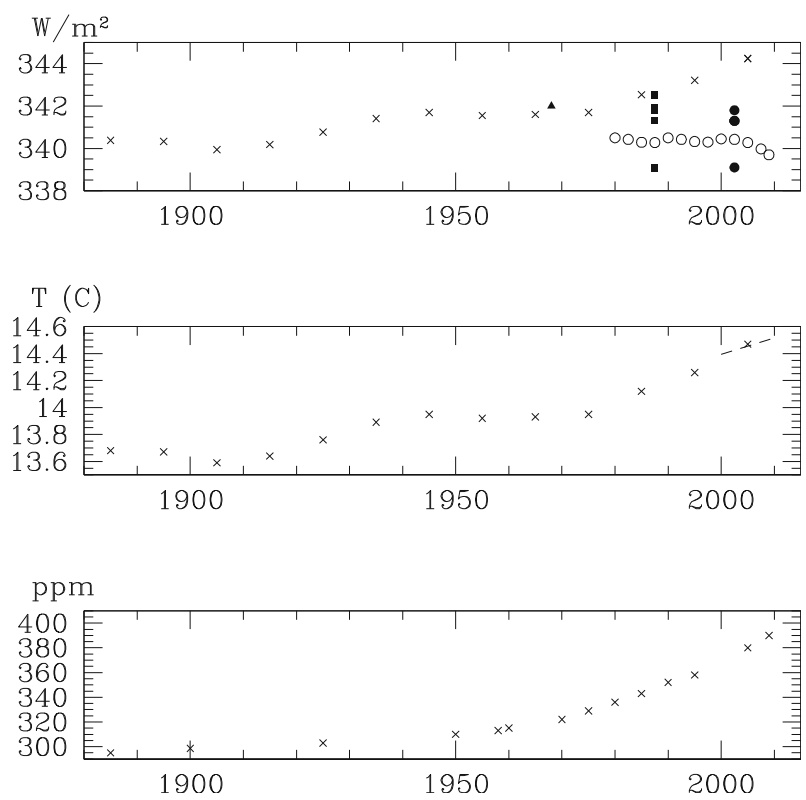

Fig. 1 Upper panel-The fluxes $\mathcal{F}_{\mathrm{S}} / 4$. Crosses $340.4+3 \sigma \Delta T / T^{3} \mathrm{Wm}^{-2}, T=287 \mathrm{~K}$ computed from the growth $\Delta T$ of the temperature in the middle panel. Triangle (Unsöld, 1968), filled squares and circles fluxes from the observations of the satellites ERBE and CERES, respectively, [the outlier points at the bottom are from the Japanese reanalysis (Trenberth et al. 2009)], circles fluxes from the satellites ACRYM etc. covering three solar cycles (Solanki and Unruh 2013; Solanki et al. 2013). Middle panel Crosses decadal global combined surface-air temperature $\left({ }^{\circ} \mathrm{C}\right.$ ) over land and sea-surface, 1880-2010 (Jarraud 2013), these are the input data to the crosses in the upper panel. Dashed line linear regression to the annual averaged temperature, 2000-2010. Lower panel-The atmospheric $\mathrm{CO}_{2}$ concentration (Jacob 1999; Jarraud 2013.) 
Two uncertain and hardly discussed components must be qualitatively mentioned here for the sake of completeness which are connected with the celestial mechanics. The largest annual periodic variation $\Delta \mathcal{F}_{\mathrm{S}} / \mathcal{F}_{\mathrm{S}}=|-2 \Delta d / d| \approx 0.064\left(\Delta F_{\mathrm{S}}=87 \mathrm{Wm}^{-2}\right)$ is the consequence of the eccentricity 0.016 of the orbit of the Earth. This exceeds the observed temporal variations of $\mathcal{F}_{\mathrm{S}}$ by almost two orders of magnitude. Another less discussed component is the consequence of the tilt of the rotation axis of the Earth with a precession period $\approx 26,000$ years. This effect is coupled to the precession of the orbit with a period $\approx 113,000$ years. The periods are very close to a 9:2 resonance. These hardly discussed components can be coupled to possible climate changes over $\approx 230,000$ years by the different absorbing capacity of the TSI in the Northern and Southern hemisphere because of the higher absorbing capacity of the oceans (Granopolski et al. 2016).

\section{Flux propagation in an air column, the radiative contribution to the greenhouse effect}

The stationary transfer of specific intensity $I_{\lambda}\left(\theta, \tau_{\lambda}\right)$ of wavelength $\lambda$ is described in the EAC approximation by the

$$
\cos \theta \frac{\mathrm{d} I_{\lambda}\left(\theta, \tau_{\lambda}\right)}{\mathrm{d} \tau_{\lambda}}=I_{\lambda}\left(\theta, \tau_{\lambda}\right)-S_{\lambda}\left(\theta, \tau_{\lambda}\right)
$$

monochromatic transfer equation where $\theta$ is the polar angle in a spherical reference system with axis $z$ perpendicular to the surface, $z=0$ at the bottom of the atmosphere (BOA). Isotropy is assumed in the azimuth. The plan-parallel approximation is involved in (7), it is permissible because of the small geometrical thickness of the atmosphere compared to the planetary radius. $S_{\lambda}\left(\theta, \tau_{\lambda}\right)$ is the source function at the optical depth

$$
\tau_{\lambda}(z)=\tau_{\lambda}(z=0)-\int_{0}^{z} \mathrm{~d} z^{\prime} \kappa_{\lambda}\left(z^{\prime}\right), \quad \mathrm{d} \tau_{\lambda}=-\kappa_{\lambda} \mathrm{d} z
$$

(Mihalas 1978), (the units of $I_{\lambda}$ and $S_{\lambda}$ are $\mathrm{Wm}^{-2} \operatorname{sterad}^{-1}$ ), $\kappa_{\lambda}=\kappa_{\lambda, \mathrm{at}} n(z)$ is the monochromatic absorption coefficient which is the product of the photon absorbing or scattering cross section $\kappa_{\lambda \text {,at }}$ and number density $n(z)$ of an atmospheric component at height $z$, their dimensions are $\mathrm{m}^{2}$ and $\mathrm{m}^{-3}$, respectively. $\tau_{\lambda}=0$, at the TOA.

The line broadening in a planetary atmosphere produces a depth-dependent Dopplerprofile. It can be approximated by a rectangle $P(\lambda)$ of Doppler-width belonging to a local or average temperature in the air column. $P(\lambda)$ must be normalized to unity in the wavelength interval of the line, $\kappa_{\lambda, \text { at }}$ is an atomphysical quantity, it does not depend on geometrical height. The optical depth at the TOA depends therefore only on the $N=$ $\int \mathrm{d} z^{\prime} n\left(z^{\prime}\right)$ column density of the absorbent:

$$
\tau_{\lambda}=\kappa_{\lambda, \mathrm{at}} P(\lambda) N .
$$

(At the see level: $N_{\mathrm{CO}_{2}}=5.47 \times 10^{21} \mathrm{~cm}^{-2}$ for $400 \mathrm{ppm}$ concentration, $N_{\mathrm{CH}_{4}}=6.80 \times$ $10^{19} \mathrm{~cm}^{-2}$ for $1.808 \mathrm{ppm}$.)

The simplification (9) can be allowed because the spectral irradiation of the air column is practically constant within the line profile. It is useful because the target of this study is to compute the change of the emerging integrated flux at the TOA as a function of $N$. (The 
goals of the computation are neither the absolute value of the flux nor a line profile at the TOA.)

The intervals $0 \leq \theta \leq \pi / 2$ and $\pi / 2 \leq \theta \leq \pi$ are for the outward and inward fluxes $\mathcal{F}_{\lambda}^{(+),(-)}\left(\tau_{\lambda}\right)=2 \pi \int_{0, \pi / 2}^{\pi / 2, \pi} \mathrm{d} \theta \sin \theta \cos \theta I_{\lambda}\left(\theta, \tau_{\lambda}\right)$. The Eddington approximation can be applied for the upward and downward fluxes separately, that is $\partial, I_{\lambda}^{(+)}{ }^{(-)}\left(\theta, \tau_{\lambda}\right) / \partial \theta=0$ within the intervals $0 \leq \theta \leq \pi / 2$ and $\pi / 2 \leq \theta \leq \pi$, respectively, however, $\mathcal{F}_{\lambda}^{(+)} \neq \mathcal{F}_{\lambda}^{(-)}$. A multiplication of (7) by $2 \pi \sin \theta \cos \theta$ and an integration over $\left.\theta\right|_{0} ^{\pi / 2}$ and $\left.\theta\right|_{\pi / 2} ^{\pi}$ leads to the equations

$$
\begin{gathered}
\frac{\mathrm{d} \mathcal{F}_{\lambda}^{(+)}}{\mathrm{d} \tau_{\lambda}^{\prime}}=\frac{3}{2}\left[\mathcal{F}_{\lambda}^{(+)}-S_{\lambda}^{(+)}\right], \\
\frac{\mathrm{d} \mathcal{F}_{\lambda}^{(-)}}{\mathrm{d} \tau_{\lambda}^{\prime}}=-\frac{3}{2}\left[\mathcal{F}_{\lambda}^{(-)}-S_{\lambda}^{(-)}\right] .
\end{gathered}
$$

describing the propagation of flux in the upward and downward directions.

The first term on the right hand side of (10) and (11) accounts for the monochromatic absorption, the source functions $S_{\lambda}^{(+)}, S_{\lambda}^{(-)}$describe how the absorbed radiation is reemitted. There are three relevant elementary processes for the reemission.

Coherent scatter is an elastic scatter of the photons from the microphysical point of view, the wavelength of the absorbed and emitted photon is identical. The source function is in this case $S_{\lambda}^{(+),(-)}=\mathcal{F}_{\lambda}^{(+),(-)} / 2$. The upward monochromatic flux is at optical depth $\tau_{\lambda} \leq \tau_{\lambda}^{\prime} \leq 0$

$$
\mathcal{F}_{\lambda}^{(+)}\left(\tau_{\lambda}^{\prime}\right)=\mathcal{F}_{\lambda}^{(+)}\left(\tau_{\lambda}\right) \exp \left\{-3\left(\tau_{\lambda}-\tau_{\lambda}^{\prime}\right) / 4\right\}
$$

from the integration of (10) where $\mathcal{F}_{\lambda}^{(+)}\left(\tau_{\lambda}\right)$ is the (infrared) upward flux at the BOA, that is at $\tau_{\lambda}(z=0)$. A downward (infrared) flux $1-\mathcal{F}_{\lambda}^{(+)}\left(\tau_{\lambda}^{\prime}\right)$ is present in the EAC. Their sum is constant. These formulae describe the inward (optical) flux if ${ }^{(+)}$is replaced by ${ }^{(-)}$and $\mathcal{F}_{\lambda}^{(-)}\left(\tau_{\lambda}=0\right)$ denotes the flux of the spectral solar irradiance (SSI) at the TOA. The incoherent scatter (Mihalas 1978) can formally be merged in the coherent scatter, it needs no separate treatment from the point of view of flux propagation.

Extinction of the photons of wavelength $\lambda$ is expressed in terms of source function by $S_{\lambda}^{(+),(-)}=0$ : the absorbed part from the monochromatic flux $\mathcal{F}_{\lambda}$ vanishes, it is transferred into another degree of freedom, e.g. to macroscopic or microscopic kinetic energy (winds or thermalization etc.) or the absorbed flux of wavelength $\lambda$ appears at another wavelength $\lambda^{\prime}$. This latter process can be expressed mathematically by $S_{\lambda^{\prime}}^{(+),(-)} \neq 0$.

The extinction is important in the optical wavelengths when optical flux is converted to infrared one. The flux from the SSI is at $\tau_{\lambda}^{\prime}$

$$
\mathcal{F}_{\lambda}^{(-)}\left(\tau_{\lambda}^{\prime}\right)=\mathcal{F}_{\lambda}^{(-)}\left(\tau_{\lambda}=0\right) \exp \left\{-3 \tau_{\lambda}^{\prime} / 2\right\}
$$

True absorption is a process in a planetary atmosphere if the gas and the radiation are in approximate equilibrium, that is, the conditions are close to LTE. This holds for the upward flux in the infrared domain: the monochromatic infrared irradiation $\mathcal{F}^{(+)}\left(\tau_{\lambda}\right)$ is approximately the Planck-distribution (5) at BOA (Beuttner and Kern 1965) and (5) can be 
assumed above the BOA for the whole interval $z\left(\tau_{\lambda}^{\prime}\right), 0 \leq \tau_{\lambda}^{\prime} \leq \tau_{\lambda}$ with local temperature $T(z)$. This is expressed by using (5) in $S_{\lambda}(z)=-S_{\lambda}(z)=\mathcal{F}_{\lambda}^{(+)}[T(z)]$. The local temperature is equal in first approximation to the local effective temperature of the irradiating flux: $T(z)=T_{\mathrm{e}}(z),+$ corrections .

\subsection{The OLR}

The OLR is the integral over the appropriate infrared wavelengths:

$$
\mathcal{F}^{(+)}\left(\tau_{\lambda}^{\prime}=0\right)=\int \mathrm{d} \lambda \mathcal{F}_{\lambda}^{(+)}\left(\tau_{\lambda}\right) \exp \left\{-K_{\lambda}\right\}
$$

where $K_{\lambda}=k \tau_{\lambda}=k \kappa_{\lambda, \text { at }} P(\lambda) N$ and $k=\frac{3}{4}$ or $k=\frac{3}{2}$ are for the coherent scatter or extinction, respectively. The atmosphere is optically thin in a line if $K_{\lambda} \ll 1$. Remarkable is that $N$ is common for an atmospheric component at any $\lambda$ and the OLR linearly depends on $N$ if $K_{\lambda} \ll 1$.

The empirical definition (1) of the greenhouse effect is expressed by

$$
\mathcal{F}_{\mathrm{G}}=\int \mathrm{d} \lambda\left[\mathcal{F}^{(+)}\left(\tau_{\lambda}\right)-\mathcal{F}^{(+)}\left(\tau_{\lambda}^{\prime}=0\right)\right]=\int \mathrm{d} \lambda \mathcal{F}^{(+)}\left(\tau_{\lambda}\right)\left[1-\exp \left\{-K_{\lambda}\right\}\right]
$$

the integration is over the infrared wavelengths. The depth dependent emittance (conversion of optical irradiation to infrared emittance, an additive term) was neglected in (15).

\subsection{The albedo}

The albedo is obtained by an integration of the reflected flux (12) from the coherent scatter

$$
a=\frac{\int \mathrm{d} \lambda \mathcal{F}_{\lambda}^{(-)}\left(\tau_{\lambda}=0\right)\left[1-\exp \left\{-3 \tau_{\lambda} / 4\right\}\right]}{\int \mathrm{d} \lambda \mathcal{F}_{\lambda}^{(-)}\left(\tau_{\lambda}=0\right)} .
$$

(The integration is over the optical wavelengths.)

\subsection{The absorbed radiation}

The absorbed upward flux is

$$
\mathcal{F}^{(+)}\left(z^{\prime}\right)=\int \mathrm{d} \lambda \mathcal{F}_{\lambda}^{(+)}(z=0)\left[1-\exp \left\{K_{\lambda}-k \kappa_{\lambda, \mathrm{at}} P(\lambda) \int_{0}^{z^{\prime}} \mathrm{d} z n(z)\right\}\right]
$$

is available to forcing of the atmosphere at a height $z^{\prime}$. It is maximal if extinction dominates the interaction of the radiation and atmospheric gases $\left(k=\frac{3}{2}\right)$, this is expected in optically thick atmospheres, e.g. in Venus. It is minimal if coherent scatter dominates $\left(k=\frac{1}{2}\right)$, that is in optically thin atmospheres. The rule is for an EAC: minimal true absorption-maximal OLR and maximal true absorption-minimal OLR. The terrestrial atmosphere is between these two limits, it is approximately optically thin in optical wavelengths while it is thick in some infrared wavelengths where true absorption is expected to dominate the radiation transfer (e.g. at $\approx 670 \pm 100 \mathrm{~cm}^{-1}$ or $\approx 1300 \mathrm{~cm}^{-1}$ for $\mathrm{CO}_{2}$ and $\mathrm{CH}_{4}$, respectively.) 


\subsection{Remark}

For actual computations, it is useful to remark that Taylor expansions can easily be constructed for the monochromatic functions (12), (13), (14), (15) and (16) in terms of $\tau_{\lambda}$. These expansions can be transformed to expansions in terms of the $\Delta N$ variation of the column density of an absorbing gas. $\Delta N$ is common for all $\lambda$.

\section{The radiative role of $\mathrm{CO}_{2}$ in the change of the greenhouse effect}

The clear sky forcing was measured by the atmospheric emitted radiance interferometer (AERI) in the decade 2000-2010 (Feldman et al. 2015): an average growth $\Delta \mathcal{F}=$ $0.2 \pm 0.065 \mathrm{Wm}^{-2}$ has been found with large seasonal ranges $0.1-0.2 \mathrm{Wm}^{-2}$. It was assumed that this growth under the cloudless sky can be attributed exclusively to the increase

$$
\Delta v=\Delta N / N=22 \mathrm{ppm}
$$

of the $\mathrm{CO}_{2}$ concentration during this period. This observational result can be used in the EAC approximation to estimate the contribution of the increasing $\mathrm{CO}_{2}$ concentration to the global present day forcing. The increment of the flux from the AERI observations must be compared to that from the Stefan-Boltzmann law if the observed increase $\Delta T$ of the temperature at the surface (middle panel in Fig. 1) is substituted for $\Delta T_{\mathrm{e}}$ in

$$
\Delta \mathcal{F}=\frac{4 \Delta T_{\mathrm{e}}}{T_{\mathrm{e}}} \mathcal{F}
$$

- The increment is $\Delta T=0.124 \pm 0.061$ from a linear regression for the decade 20002010 (dashed line in the middle panel of Fig. 1.) The growth of flux is $\Delta \mathcal{F}=$ $0.66 \pm 0.32 \mathrm{Wm}^{-2}$ from (19) if the BOA values $T_{\mathrm{e}}=287 \mathrm{~K}, \mathcal{F}=384 \mathrm{Wm}^{-2}$ are used. Dividing the increments gives that $30 \pm 15 \%$ of the growth originates from the increasing atmospheric concentration of $\mathrm{CO}_{2} .\left(\Delta \mathcal{F}=0.58 \mathrm{Wm}^{-2}\right.$ if the more recent $\mathcal{F}=320 \pm 10 \mathrm{Wcm}^{-2}$ (Stephens et al. 2012) is used in (19) resulting in the slightly higher $35 \%$ contribution of $\mathrm{CO}_{2}$.)

- An improved estimation can be obtained if the flux growth observed by AERI is extrapolated for the longer period $\approx 1750-2010$. The increment is $290 \rightarrow 400 \mathrm{ppm}$ $(\Delta v=+110 \mathrm{ppm})$ in the atmospheric concentration of $\mathrm{CO}_{2}$ from the beginning of the industrial revolution in the nineteenth century: the extrapolated result is $\Delta \mathcal{F}=1.0 \pm 0.33 \mathrm{Wm}^{-2}$. The secular increment of temperature at the BOA (Fig. 1, crosses in the middle panel) is $\Delta T_{\mathrm{e}}=0.88 \mathrm{C}$ yielding $\Delta \mathcal{F}=4.72 \mathrm{Wm}^{-2}$ from (19). $(1 \pm 0.32) / 4.72=21 \pm 7 \%$ is the contribution of the $\mathrm{CO}_{2}$ to the radiative forcing from the $\Delta v \approx 110 \mathrm{ppm}$ increment.

\section{Discussion}

\subsection{Conversion of flux to temperature}

Temperature is an important quantity for the meteorology or climatology. It can be measured easily, large data sets are available at a great number of geographical points on 
the land. The homogenization and derivation of global temperature averages form a nontrivial task. Relevant quantity is the flux for a physical interpretation of climatic processes. Conversion between temperature and flux can be done if the interaction of flux (power/surface) with planetary surface and atmosphere is described properly: the average energy density of the planetary matter is calculated from the balance of the input and output power density. The factors depend on geographical position and diurnal variation.

The effective temperature $T_{\mathrm{e}}$ of the radiation can be derived from the flux by the StefanBoltzmann law, however, the interaction of the radiation and matter takes place under conditions that are far from LTE. Only a part of the radiative flux is converted to internal energy of the gas to which temperature can finally be attached unequivocally. Equation (3) is a global power balance, dividing it by the terrestrial surface enables to convert it to flux data and to use the EAC approximation.

Comparing the observed flux and temperature data for 1980-2010 (Fig. 1) reveal that the variation of the TSI is excluded as a cause for the increase of the global temperature in the twentieth century.

The concentration of $\mathrm{CO}_{2}$ is monotonically increasing in the twentieth century (Fig. 1, lower panel) while $\Delta T$ shows undulatory behaviour with a characteristic time $\approx 40$ years: the rise between 1905 and 1935 is followed by the stagnation $\Delta T \approx 0$ between 1935 and 1975 and a more intensive rise from 1975 . The slope is $\approx 0.157 \mathrm{C} /$ decade between 1980 and 2000, while it decreased to $0.124 \pm 0.061 \mathrm{C} /$ decade in 2001-2010 (leading to speculations where is the missing heat, Tollefson 2014.) A resolution of this problem can be that the storing capacity of the atmosphere follows the radiative input with some delay, e. g. because of the role of the heat reservoirs represented by the solid surface and oceans. These have a much larger capacity for storing heat than the atmosphere merely. Another factor for the non-simultaneous behaviour is that the radiation and the atmosphere are not in LTE (Mihalas 1978.)

A hardly examined radiative factor of the non-concordance of TSI and global warming can be the spectral difference of the atmospheric and black-body radiation: the derived global combined surface-air temperature is not the effective temperature: $T(z=0) \neq T_{\mathrm{e}}(z=0)$. However, $\Delta T_{\mathrm{e}}=\Delta T$ is a more reliable input in the Stefan-Boltzmann law as an estimation of first step because its basis, the Planck spectral distribution of the radiation is a good first approximation for emission into the atmosphere. An argument directing attention to this problem is that the proper zero point of the crosses in the upper panel would be $384.8 \mathrm{Wm}^{-2}$ belonging to $T=\mathrm{K} 287$ at the BOA (Jarraud 2013), not the applied $340.4 \mathrm{Wm}^{-2}$ belonging to the lower $T_{\mathrm{e}}=278.3 \mathrm{~K}$ derived from the TSI at the TOA (Table 1 ) or the even lower $\approx 320 \mathrm{Wm}^{-2}$ (Stephens et al. 2012).

Nevertheless, a much better approximation can be expected for the secular change $\Delta \mathcal{F}$ if merely the changes $\Delta T$ from the global combined surface-air temperature curves (Fig. 1) are used in the EAC approximation as $\Delta T_{\mathrm{e}}$ in (19). A comparison of $\Delta \mathcal{F}$ derived from $\Delta T(z=0)=0.88 \pm 0.1 \mathrm{C}$ and the AERI measurements is permissible since the changes could be estimated with better relevance even if the zero point of $\mathcal{F}$ was less certain. The result of this comparison is that $30 \pm 15 \%$ has been found for the contribution of increasing $\mathrm{CO}_{2}$ concentration under clear-sky conditions. (It must be remarked that the reported clear-sky measurements with the AERI merge the effect of $\mathrm{CO}_{2}$ and the other greenhouse gases (e.g. $\mathrm{CH}_{4}, \mathrm{~N}_{2} \mathrm{O}$ ), etc. (Jacob 1999), their concentration changed during the period 2000-2010 as well as that of $\mathrm{CO}_{2}$ (Jarraud 2013).

The more reliable percentage $21 \pm 7$ as the effect of human industrial activities since $\approx 1750$ is obtained if the observed $\Delta \mathcal{F}$ of the AERI measurements is extrapolated for this 
whole period and it is compared to the temperature data for 120 years (Jarraud 2013). (The error $7 \%$ was derived from the error of the flux measurement. The inclusion of the estimated error of $T_{\mathrm{e}}$ would increase it to $\approx 9-10 \%$.) This extrapolation is appropriate if the atmosphere is optically thin for the greenhouse gases, that is the higher terms of the expansion (14) are negligible: $K_{\lambda} \ll 1$ for all relevant infrared wavelengths. This condition would only be satisfied if $\kappa_{\lambda, \text { at }} \ll 5 \times 10^{-19} \mathrm{~cm}^{2}$ for the photon scattering cross section of the infrared transitions in the spectrum $(\lambda=5-50$ micron $)$ of $\mathrm{CO}_{2}$ as follows from (9). The condition is not satisfied at see level e.g. in the centre of the $\mathrm{CO}_{2}$ bands ( $\approx 16$ micron.) However, the obtained $21 \%$ is a good starting point to more refined computations because the Taylor expansion of the fluxes in terms of $\Delta v(=\Delta N / N)$ converges better.

Raval and Ramanathan (1989) estimated that the greenhouse effect of a doubling of $\mathrm{CO}_{2}$ is $\Delta \mathcal{F}=4 \mathrm{Wm}^{-2}(+16 \mathrm{~W}$ in TSI) giving the averaged slope $\Delta \mathcal{F} / \Delta v=1.38 \times$ $10^{-2} \mathrm{Wm}^{-2} / \mathrm{ppm}$ for the whole period from 1750 . More recent radiative transfer models involving better data of the radiative properties of $\mathrm{CO}_{2}$ gave the slope of the global mean radiative forcing $\Delta \mathcal{F} / \Delta v=(1.66 \pm 0.17) \times 10^{-2} \mathrm{Wm}^{-2} / \mathrm{ppm}$ at the tropopause (Feldman et al. 2015) as a consequence of the growth of $\mathrm{CO}_{2}$ concentration since 1750. Comparing these data to the increase of flux derived from the secular increase of temperature at the $\mathrm{BOA}$ (Fig. 1, middle panel) gives that some $\approx 21 \pm 7 \%$ is the contribution of the $\mathrm{CO}_{2}$ to the radiative forcing for the $\Delta v \approx 110 \mathrm{ppm}$ rise. However, this is significantly higher than $\Delta \mathcal{F} / \Delta v=(9.09 \pm 0.27) \times 10^{-3} \mathrm{Wm}^{-2} / \mathrm{ppm}$ extrapolated from the AERI measurements. The discrepancy suggests that the theoretically computed increment of the flux from the rising concentration of the greenhouse gas $\mathrm{CO}_{2}$ may need a revision.

The Formulae (14) and (16) offer an opportunity to estimate the quantitative dependence of greenhouse forcing as a function of the (change) of the atmospheric components. Remarkable is the primary dependence on column density and the almost perfect independence on the temperature represented by $P(\lambda)$ in the EAC.

\section{Conclusions}

In EAC approximation, the empirical definition of the atmospheric greenhouse effect has been formulated in fluxes. Flux is extensive quantity in the theory of the radiative transfer. Problems to convert the fluxes to more accessible intensive quantity temperature have only been sketched.

A comparison of observed forcing between 2000 and 2010 and the integrated fluxes from the terrestrial surface temperatures derived by the Stefan-Boltzmann-law has shown that the rise of the atmospheric concentration of $\mathrm{CO}_{2}$ from the start of the industrial revolution has contributed to the radiative forcing of the atmosphere by some $21 \pm 7 \%$. This percentage cannot exceed $39 \%$ even if the most recent LBL calculations are applied to compute the forcing, however, they probably need a revision to be consistent with the AERI observations. The discrepancy between the percentage of forcing from the empirical basis and the theoretical calculations indicates a need to reconsider the theoretical calculations concerning the radiative role of the increasing $\mathrm{CO}_{2}$ concentration. A formalism of this has been described in this paper.

The main result has been that the radiative forcing from the growth of concentration of the non-condensing greenhouse gas $\mathrm{CO}_{2}$, released by the industrial activity in the twentieth century, contributed to the observed forcing up to $\approx 20-30 \%$. The presented results from treating the radiative flux propagation in the atmosphere have left open the question for the 
source of the remnant global forcing $\approx 70-80 \%$. On speculation level the conclusion is probable that the relaxation times of the interaction of radiation and atmospheric gases under non-LTE conditions, the effect of the aerosols (produced by industrial activity and natural processes) and water (in non-condensed or condensed form) seem to be more important regulators of the observed global warming in the twentieth century. Furthermore, long term power release of accumulated heat from the oceans and solid surface can contribute to the $\approx 70-80 \%$.

Acknowledgments The author is grateful to D. Drahos, L. Haszpra, L. van Driel-Gesztelyi I. Késmárky for many discussions and technical help. Comments of anonymous referees are gratefully acknowledged.

\section{References}

Beuttner KJK, Kern CD (1965) The determination of infrared emissivities of terrestrial surfaces. J Geophys Res 70:1329

Feldman DR, Collins WD, Gero PJ, Torn MS, Mlawer EJ, Shippert TR (2015) Observational determination of surface radiative forcing by CO2 from 2000 to 2010. Nature 519:339

Granopolski A, Winkelmann R, Schellnhuber HJ (2016) Critical insolation-CO2 relation for diagnosing past and future glacial inception. Nature 529:200

International Energy Agency (2014) Report

Jacob D (1999) Introduction to atmospheric chemistry. Princeton University Press, Princeton

Jarraud M (2013) World Meteorological Organization, WMO-No. 1119

Lowrie W (2007) Fundamentals of geophysics, 2nd edn. Cambridge University Press, Cambridge

Mihalas D (1978) Stellar atmospheres. W. H. Freeman and Co, San Francisco

Raval A, Ramanathan V (1989) Observational determination of the greenhouse effect. Nature 342:758

Solanki SK, Unruh YC (2013) Solar irradiance variability. Astr Nach 334:145

Solanki SK, Krivova NA, Haigh JD (2013) Solar irradiance variability and climate. Ann Rev Astron Astrophys 51:311

Stephens GL et al (2012) An update on Earth's energy balance in light of the latest global observations. Nat Geosci 5:691

Tollefson J (2014) Climate change: the case of the missing heat. Nature 505:276

Trenberth KE, Fasullo JT, Kiehl J (2009) Earth's global energy budget. Bull Am Meteor Soc 90:311

Trenberth KE, Fasullo JT, Balmaseda MA (2014) Earth's energy imbalance. J Clim 27:3129

Unsöld A (1968) Physik der Sternatmospharen. Springer, Berlin 